\title{
BROADBAND OBSERVATIONS OF RADIO EMISSION OF FLARE STARS
}

\author{
D. V. Mukha*, A. A. Konovalenko*, H. O. Rucker ${ }^{\dagger}$, V. L. Kolyadin*, \\ A. S. Nabatov*, V.V. Zakharenko*
}

\begin{abstract}
Studies of flare stars at radio waves are an important task of modern radio astronomy. They are connected with investigations of the physics of the stellar wind and stellar-planetary interrelations including habitable zones. One special interest is the search for radio emission bursts at extremely low frequencies (decameter wavelength). According to the analogy with the solar bursts the low frequency stellar bursts radio emission are arising in the outer stellar wind. Simultaneous observations at low frequencies and higher frequencies (from centimeter to meter wavelengths) are important also for the improvement of the detection reliability as well as for the phenomenology studies. We demonstrate the possibility to observe flare star bursts in the decametric radio band with the 70-meter dish radio telescope RT-70 (Evpatoria, Ukraine).
\end{abstract}

\section{Introduction}

There is a strong interest in the study of the UV Cet type active (flare) stars across the whole electromagnetic spectrum. It is an important investigation from the point of view of their representative set as the galactic population as well as for the stellar-planetary interrelations problems including the analysis of the habitable zone [Lammer et al., 2009].

In the radio domain flare stars observations have been performed during the last few decades. There are many positive results of the burst detection at centimeter-decimeter wavelengths [Bastian, 1990; Osten and Bastian, 2006] by using the VLA, Arecibo, Effelsberg or the Jodrell Bank antennas. The flare stars EV Lac, AD Leo, YZ Cmi, UV Cet were the main targets of the investigations at the L,C-band frequencies. Flux densities of the bursts are up to 1 Jy, lifetimes of about tens of minutes (with some fine structure). There is a low correlation of radio bursts with optical and X-ray flares with some delay.

\footnotetext{
* Institute of Radio Astronomy, Ukrainian Academy of Sciences, Chervonopraporna 4, 61002, Kharkiv, Ukraine

$\dagger$ Space Research Institute, Austrian Academy of Sciences, Schmiedlstrasse 6, A-8042 Graz, Austria
} 
Special interest exists in the search of flare stars radio emission at meter-decameter wavelengths. A probable similarity with the Sun (at least with the III- and II-Type Solar bursts [Melnik et al., 2004]) suggests that this radio emission must arise at farther distances from the star. This gives the possibility to perform outer stellar wind diagnostics. There were many attempts to detect the radio emission bursts at decameter wavelength by UTR-2 radio telescope but the results are not definitive. There is only episodical bursts detection at decameter wavelength by using the UTR-2 radio telescope [Abranin et al., 2001].

Few years ago an intensive observational program for the search of the flare stars radio emission bursts was started at decameter wavelengths $(10-30 \mathrm{MHz})$ [Konovalenko et al., 2008]. The observations are carried out by using of the world largest decameter array UTR-2 [Braude et al., 1978; Konovalenko, 2000]. Many radio emission bursts for ADLeo and EVLac were detected. But for the improvement of the detection reliability we need to implement an additional signal identification criteria. One of them is the simultaneous observation at higher frequencies, for example, in the decimeter wavelength. The preliminary results of flare stars radio emission observations in this radio domain are presented in this paper.

\section{Instrumentation}

We used the 70-meter dish radio telescope RT-70 (Evpatoria, National Space Agency of Ukraine) (Figure 1). This antenna is used in many radio astronomy programs and space projects [Konovalenko et al., 2005]. The parameters of the radio telescope are presented in Table 1.
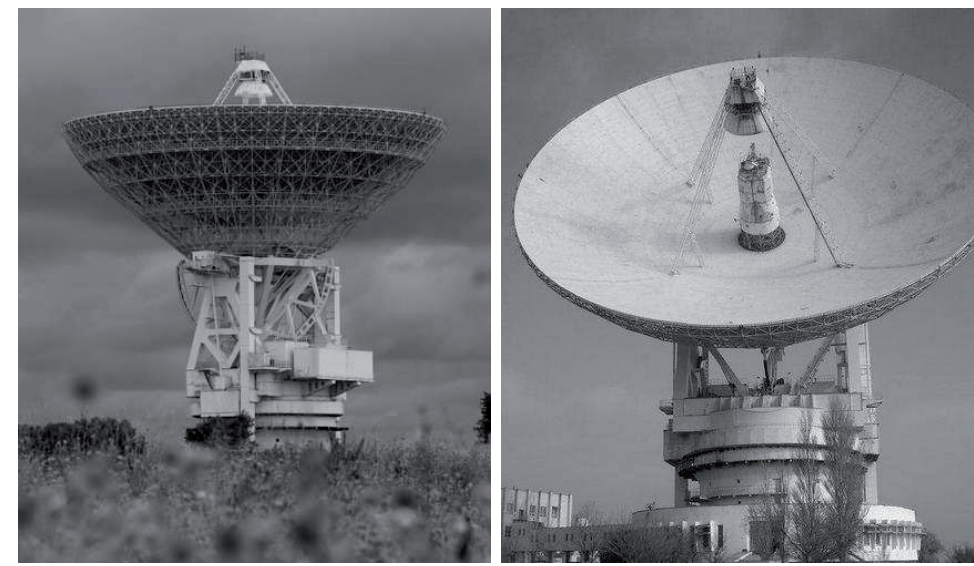

Figure 1: RT-70 Radio Telescope, Evpatoria

The L-band $(1.6 \mathrm{GHz})$ with two circular polarizations and two cryogenic receivers was implemented. The beam width is 9 angular minutes, the system temperature is $\sim 40 \mathrm{~K}$, and the effective area is near $2200 \mathrm{~m}^{2}$. This gives a minimum detectable flux density 
of about $\sim 100 \mathrm{mJy}$. The time and frequency resolutions of the backend facility are 2,5 seconds and $100 \mathrm{kHz}$, respectively which corresponds to other stations of the same class. A Wave-form pulsar receiver has been used for the registration and data storage with a bandwidth of $7 \mathrm{MHz}$ and a central frequency of $1666 \mathrm{MHz}$ [Zakharenko et al., 2007]. During the Fourier transform of the wave-form signal time and frequency resolutions were selected by the smoothing in time and frequency.

Table 1: RT-70 work band parameters; HPBW= half power bandwith in Kelvin

\begin{tabular}{|c|c|c|c|}
\hline Band & $\begin{array}{c}\text { Bandwidth } \\
{[\mathrm{MHz}]}\end{array}$ & HPBW & $\begin{array}{c}\text { Noise } \\
\text { Temperature }\end{array}$ \\
\hline $\mathrm{P}-92 \mathrm{~cm}$ & $321.5-328.5$ & $60 '$ & 250 \\
$\mathrm{~L}-18 \mathrm{~cm}$ & $1350-1720$ & $9^{\prime} 20^{\prime \prime}$ & 40 \\
$\mathrm{~S}-13 \mathrm{~cm}$ & $2150-2490$ & $6^{\prime} 45^{\prime \prime}$ & 100 \\
$\mathrm{C}-6 \mathrm{~cm}$ & $4600-5060$ & $3^{\prime} 10^{\prime \prime}$ & 45 \\
$\mathrm{X}-3.5 \mathrm{~cm}$ & $8180-8680$ & $1^{\prime} 45^{\prime \prime}$ & 45 \\
$\mathrm{~K}-1.35 \mathrm{~cm}$ & $18,000-26,000$ & $43^{\prime \prime}$ & - \\
\hline
\end{tabular}

\section{Observations and data processing}

Two of the most active stars AD Leo and EV Lac have been selected for observations. Table 2 shows the observational sessions and the time of observations.

Table 2: Observations

\begin{tabular}{|c|c|c|c|}
\hline No. & Session time & Source & $\begin{array}{c}\text { Observation time } \\
{[\text { hour] }}\end{array}$ \\
\hline 1 & Aug, 27 - Sep, 3 2008 & EV Lac & 64 \\
2 & Feb, 16- Feb, 22 2009 & AD Leo & 30 \\
3 & Aug, 25 - Sep, 3 2009 & EV Lac & 60 \\
4 & Mar, 1 - Mar, 5 2010 & AD Leo & 45 \\
5 & Aug, 26-Sep, 3 2010 & EV Lac & 72 \\
\hline
\end{tabular}

The observations of the flare stars were carried out during $\sim 6$ hours every day for each observational session. Every two hours we observed reference radio sources for system checking and calibration. It was every two hours during the session. At the beginning and end of each session we used 3c84, 3c147 and 3c234 radio sources for calibrations. Figure 2 illustrates the transit of $3 \mathrm{c} 84$ through the antenna beam and one point of the sky without strong radio sources. Figures 3-5 show the dynamic spectra for AD Leo with presence of the burst activity. The time resolution and frequency resolution of the dynamic spectra are averaged to $2.43 \mathrm{~s}$ and $14 \mathrm{kHz}$ respectively. The plots at the bottom of each figure show profiles of the dynamic spectra at the central frequency of $1666 \mathrm{MHz}$ (vertical axies are in Jy). As one can see there is an evident excess of the events level over the noise fluctuations. Figure 6 illustrates dynamic spectra for the EV Lac with $600 \mathrm{~ms}$ 
time resolution and $14 \mathrm{kHz}$ frequency resolution. As one can see from the Figures 3-6 the maximum intensity of the bursts is about $0.5 \mathrm{Jy}$. The duration of the episodes is $10-20$ minutes. The evident fine structures are at the scale of about $1 \mathrm{~min}$.

The fine structure components on the dynamic spectra have some negative and positive drifts. According to our measurements schedule the activity rate is near one strong episode per 50 hours. It is also possible to implement the next step of the processing including averaging of the data, comparison with another frequency ranges.
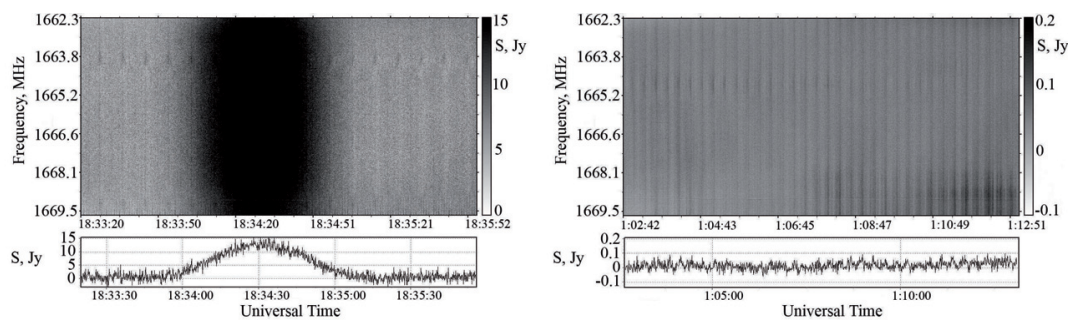

Figure 2: Dynamic spectrum of calibration source 3c84 and dynamic spectrum of sky without radio sources at left-circularly polarization. The bottom plots are the profiles of the dynamic spectra at central frequency $1666 \mathrm{MHz}$; the vertical axies are in $J y$, the horizontal axies are universal time (hours, minutes, seconds).
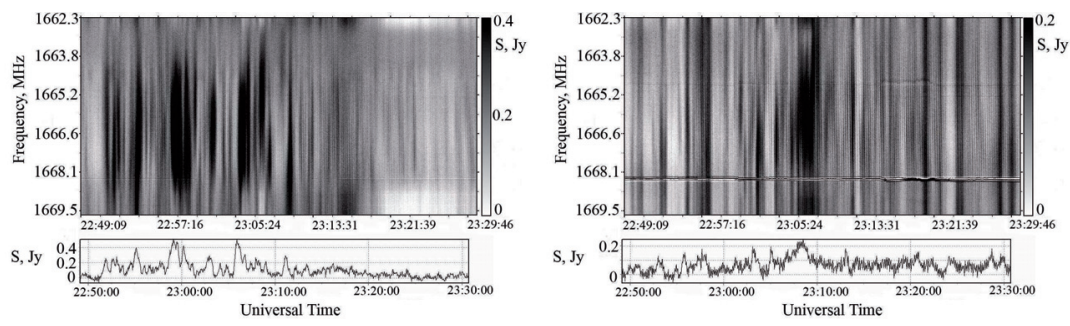

Figure 3: Dynamic spectra of the radio burst observed on AD Leo at left-circularly polarization (left panel) and right-circularly polarization (right panel) on 19 February 2009.
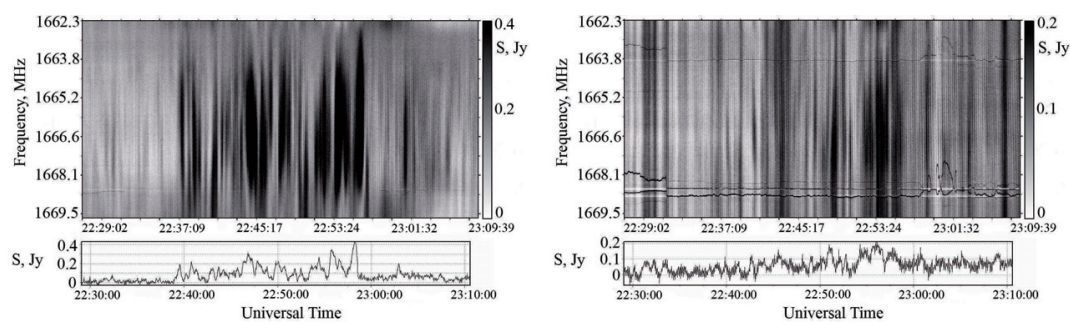

Figure 4: Dynamic spectra of the radio burst observed on AD Leo at left-circularly polarization (left panel) and right-circularly polarization (right panel) on 22 February 2009. 

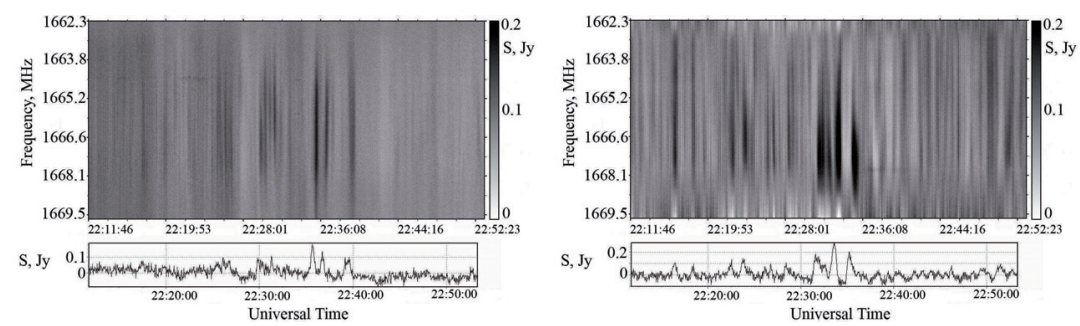

Figure 5: Dynamic spectra of the radio burst observed on AD Leo at left-circularly polarization (left panel) and right-circularly polarization (right panel) on 02 March 2010.
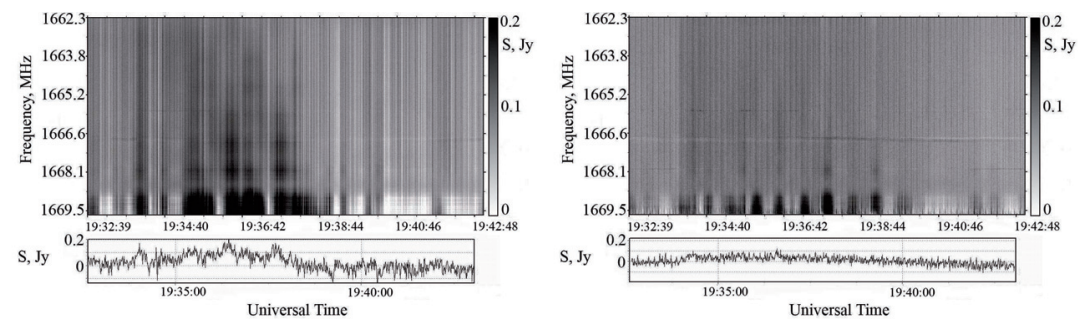

Figure 6: Dynamic spectra of the radio burst observed on EV Lac at left-circularly polarization (left panel) and right-circularly polarization (right panel) on 26 August 2009.

\section{Conclusions}

We illustrate the possibility of flare stars bursts radio emission observations by using the RT-70 radio telescope (Evpatoria) at decimeter wavelengths. The parameters of the observed bursts are in agreements with previous similar observations [Bastian, 1990; Osten and Bastian, 2006]. The maximum intensity is about $500 \mathrm{mJy}$, lifetime is near to 20 minutes, and detectable fine structure is about tens of seconds. These experiments confirm the high importance of simultaneous observations with another radio domain, at first, at decameter wavelength. It will improve the reliability of the data and physical analysis of these phenomena in a broad frequency range. The next step of this study is connected with the careful comparison of the data with the existing UTR-2 radio telescope results as well as with new simultaneous experiments.

\section{References}

Abranin, E. P., I. Yu. Alekseev, L. L. Bazelyan, A. I. Brazhenko, R. E. Gershberg, V. N. Lisachenko, and N. I. Sharkhovskaja, Decameter radioemission from red dwarf flare stars, Radio Physics and Radio Astronomy (in Russian), 6, 89-102, 2001.

Bastian, T. S., Radio emission from flare stars, Solar Phys., 130, 265-294, 1990. 
Braude, S. Ya, A. V. Megn, and L. G. Sodin, The UTR-2 radiotelescope at decameter wavelengths, Antennas (in Russian), 26, 3-15, 1978.

Konovalenko, A. A., Ukraine decameter wave radio astronomy systems and their perspectives, Radio Astronomy at Long Wavelengths, Geophysical Monograph Series, 119, 311-319, 2000.

Konovalenko, A. A., I. S. Falkovich, A. S. Nabatov, O. M. Ulyanov, L. P. Suslov, K. vant Klooster, and A. G. Petrenko, Status of the 70 meter antenna in Evpatoria, 28-th ESA Antenna Workshop, ESTEC, 589-596, 2005.

Konovalenko, A. A., et al. (16 co-authors), The search of planetary and stellar decameter wavelength radio emissions by the use of the world largest radio telescope, Geophysical Research Abstracts, EGU General Assembly, Austria, 10, 2008.

Lammer, H., et al. (16 co-authors), What makes a planet habitable? The Astronomy and Astrophysics Review, 17, 181-249, 2009.

Melnik, V. N., A. A. Konovalenko, H. O. Rucker, A. A. Stanislavsky, E. P. Abranin, A. Lecacheux, G. Mann, A. Warmuth, V. V. Zaitsev, M. Y. Boudjada, V. V. Dorovskyy, V. V. Zaharenko, V. N. Lisachenko, and C. Rosolen, Observations of solar type II bursts at frequencies 10-30 MHz, Solar Phys., 222, 151-166, 2004.

Osten, R. A., and T.S. Bastian, Wide-Band Spectroscopy of Two Radio Bursts on AD Leonis, Astrophys. J., 637, 1016-1024, 2006.

Zakharenko, V.V., V.S. Nikolaenko, O. M. Ulyanov and R. A. Motiyenko, A High Time Resolution Receiver for Radio Emission Investigation, Radio Physics and Radio Astronomy (in Russian), 12, 233-241, 2007. 